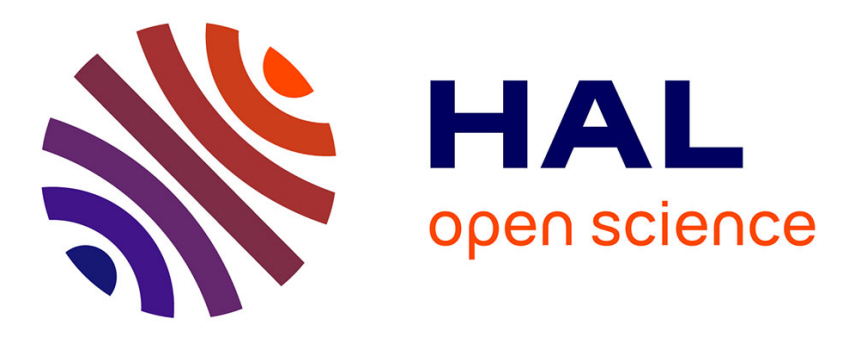

\title{
Optical near-field distributions of surface plasmon waveguide modes
}

\author{
J. C. Weeber, Y. Lacroute, Alain Dereux
}

\section{To cite this version:}

J. C. Weeber, Y. Lacroute, Alain Dereux. Optical near-field distributions of surface plasmon waveguide modes. Physical Review B: Condensed Matter and Materials Physics (1998-2015), 2003, 68 (11), pp.115401. 10.1103/PhysRevB.68.115401 . hal-00472590

\section{HAL Id: hal-00472590 \\ https://hal.science/hal-00472590}

Submitted on 12 Apr 2010

HAL is a multi-disciplinary open access archive for the deposit and dissemination of scientific research documents, whether they are published or not. The documents may come from teaching and research institutions in France or abroad, or from public or private research centers.
L'archive ouverte pluridisciplinaire HAL, est destinée au dépôt et à la diffusion de documents scientifiques de niveau recherche, publiés ou non, émanant des établissements d'enseignement et de recherche français ou étrangers, des laboratoires publics ou privés. 


\title{
Optical near-field distributions of surface plasmon waveguide modes
}

\author{
Jean-Claude Weeber, Yvon Lacroute, Alain Dereux \\ Laboratoire de Physique de l'Université de Bourgogne (UMR CNRS 5027), \\ 9 avenue A. Savary, F-21078 Dijon, France
}

(June 19, 2003)

\begin{abstract}
Thin gold stripes, featuring various widths in the micrometre range were microfabricated to obtain surface plasmon guides on a glass substrate. Each metal stripe (MS) was excited by an incident surface plasmon polariton which was itself launched on an extended thin gold film by the total internal reflection of a focused beam coming through the substrate. The optical near-field distributions of the surface plasmon modes sustained by the stripes were then recorded using a Photon Scanning Tunneling Microscope (PSTM). For a fixed frequency of the incident light, these field distributions are found to depend on the widths of the stripes. We first provide an experimental study of the various order modes which arise as a function of the decreasing width. Specifically, we show that the lateral confinement of a MS surface plasmon modes on is not related to a reflection of the $\mathrm{SP}$ on the edges of the stripe. On the basis of PSTM images recorded over a a gold thin film step discontinuity, we show that the metal stripe plasmon modes are hybrid modes created by the coupling of interface and boundary modes. Using MS of various thicknesses, we finally demonstrate that, similarly to the symmetric mode of an
\end{abstract}


extended metal thin film bounded by different dielectric media, the field of the MS modes is mostly localized at the interface between the metal and the dielectric medium with the lowest refractive index.

PACS:

07.79.Fc Near-field scanning optical microscopes

42.79.Gn Optical waveguides and couplers

73.20.Mf Collective excitations (including plasmons

and other charge-density excitations)

78.66.-w Optical properties of specific thin films,

surfaces, and low- dimensional structures 


\section{INTRODUCTION}

At optical frequencies, a surface plasmon $(\mathrm{SP})$ is an electromagnetic mode which results from the oscillation of polarization charges at the interface between a metal and a dielectric medium [1]. SP modes are said to be bound to the metal surface, or to be non-radiative, when they display an evanescent decay in the dielectric medium. The conditions of excitations of SP are well-known on an extended thin film. By "extended thin film", we mean a film of finite thickness in the direction $z$, whose dimensions in the $x$ and $y$ directions are so large that they can be considered as infinite when compared with the incident wavelength. On the contrary, only a few data exist about SP modes sustained by thin Metal Stripes (MS) featuring micrometre widths in, for instance, the $x$ direction. Recent works indicated that MS can be used as surface plasmon guides. When buried in an homogeneous dielectric medium, theoretical estimates expect that MS can guide so called "long-range" SP modes [2,3] over several millimetres at telecommunication frequencies [4]. For a MS deposited on a dielectric substrate, the SP mode field is more confined in the metal, leading to typical propagation lengths of a few tenths of micrometers [5]. Thus, depending on their environment, MS could be of practical interest for building plasmonic devices useful to integrated optics [6] or for better controlled optical addressing of molecular structures. An appealing feature of the embodiment of MS in reduced scale optical devices is that MS allow the transport of both optical and electrical signals.

Mapping the intensity distribution of SP modes is necessary to fully characterize MS guiding properties. Specifically, such a mapping is essential to optimize the coupling between MS of various widths or between MS and other kinds of waveguides. Since the SP field decays exponentially in the direction perpendicular to the interface to which it is bounded, the observation of SP modes is conveniently achieved by a near-field optical microscope frustrating the evanescent waves. To map the electric field intensity distribution of MS surface plasmon modes, we used a Photon Scanning Tunneling Microscope (PSTM) [7,11]. We report here about the experimental observation of fine transverse modal structures in 
the intensity distribution of SP sustained by gold MS of various widths. The PSTM was already operated to demonstrate the propagation of plasmon modes along silver MS lying on a glass substrate [8], but, in this previous study, the silver samples were not suitable to observe this feature of the transverse intensity profile.

\section{EXPERIMENTAL SETUP}

A detailed description of the PSTM setup can be found in Ref. [8]. A tapered optical fiber coated with a thin layer of chromium is attached to a piezo-tube which enables to scan the tip over the sample within a range of a few tens of micrometers. The evanescent field frustrated by the probe propagates through the fiber to reach a photomultiplier tube. In this work, the PSTM images were obtained by scanning the probe at a constant height over the sample. Scanning the PSTM tip at a constant height without any force feedback control, requires a fine adjustment of the parallelism between the scanning plane and the surface of the sample. In our setup, three micrometric screws are used to tilt the sample surface with respect to the scanning plane. With such a system, a tilt smaller than $0.06^{\circ}$ along two perpendicular scanning axis can be routinely achieved by means of atomic forces microscope (AFM) measurements. A standard AFM microscope was also operated to obtain the topographic images shown in the following such that the PSTM and the AFM images pertaining to a same sample were not recorded simultaneously.

The samples appear in figure 1. They are produced by a microfabrication process involving the following steps : 1) electron-beam lithography on a ITO doped glass substrate spin-coated with polymethyl-metacrylate (PMMA); 2) thermal evaporation of a gold film; 3) lift-off of the PMMA. The resulting samples are gold structures with a thickness $55 \pm 2$ $\mathrm{nm}$ (roughness of $0.7 \mathrm{~nm}$ RMS) directly deposited on the dielectric substrate. The width of the MS ranges from 4.5 to $1.5 \mu \mathrm{m}$ by step of $0.5 \mu \mathrm{m}$. The MS are attached to an extended thin film area of about $35 \times 90 \mu \mathrm{m}^{2}$. The glass substrate is optically connected with an immersion oil to a right-angle glass prism. Coming out a Titanium-Sapphire laser with 
a wavelength in vacuum $\lambda=800 \mathrm{~nm}$, a TM polarized light beam is focused through the prism and the substrate on the extended thin film area using a 0.15 numerical aperture lens (spot diameter at half maximum $=8.6 \mu \mathrm{m}$ at the focus). Adjusting the angle of incidence to about $43^{\circ}$, a surface plasmon polariton $(\mathrm{SP})$ is launched on the extended thin film $[8,12]$. As schematically shown in figure 1(b), two conditions are necessary for the SP launched on the extended thin film to couple efficiently with the MS plasmon modes [8]. First, the

incident spot should be located in front of the MS. At the used frequency, the $\frac{1}{e^{2}}$ damping distance of a gold-air SP intensity is larger than $40 \mu \mathrm{m}$ [5]. Therefore, even if the incident spot is located close to the right edge of the thin film area, the SP reaches the stripes with a significant amplitude. Second, the plane of incidence must be aligned with the longitudinal axis of the MS.

\section{INTENSITY DISTRIBUTION OF METAL STRIPES SP MODES}

Figure 2 shows the AFM and the PSTM images of two MS respectively labelled as (3) and (4) in fig. 1(a) (respective widths: 3.5 and $3.0 \mu \mathrm{m}$ ). The PSTM images have been obtained as the MS were excited by the extended thin film SP according to the procedure described above. For both stripes, the propagation of the MS mode occurs from the top to the bottom of the images. The PSTM image of the first MS (width $w=3.5 \mu \mathrm{m}$, fig. $2(\mathrm{~b})$ ) has been recorded over its output end. The scattering of the incident SP mode at the edge of the stripe termination leads to an intense diffusion spot. Note that, to reach the MS end, the SP mode has travelled more than $45 \mu \mathrm{m}$. The interference between the incident and the back-reflected plasmon mode creates a stationary wave pattern. Thanks to the weak contrast of the interference fringes, one can see three dark lines parallel to the long axis of the MS. These longitudinal lines of the MS plasmon mode are better observed on the PSTM image recorded over the central part of the $3.0 \mu \mathrm{m}$ wide stripe (fig. $2(\mathrm{~d})$ ). In this case, only two dark lines appears along the MS. In order to study the dependence of this SP modal structure as a function the MS width, a PSTM image of each stripe shown in fig. 1 has been 
recorded. The transverse cross-cuts (along the $x$ axis) of these near-field optical images are displayed on fig. 3. The MS topographic profiles obtained by AFM (dashed lines in fig. 3) are superimposed on these cross-cuts. Since the optical and the topographic images were not recorded simultaneously, the relative position of the optical and topographic profiles have been adjusted in order to center the optical response with respect to the topography. Except for $w=1.5 \mu \mathrm{m}$, a striking feature is the strong lateral confinement of the MS surface plasmon modes within the width of the stripe [8]. With dielectric waveguides, such a lateral confinement is obtainable only for high values of index of refraction contrast between the core and the cladding [13]. The optical profile obtained for $w=4.5 \mu \mathrm{m}$ shows only shoulders with an amplitude too small to allow an accurate measurement of their inter-distance. On the contrary, four neat peaks are visible in the optical response of the MS with $w=4.0 \mu \mathrm{m}$ and $w=3.5 \mu \mathrm{m}$. However, the distance $p$ between these peaks is found to be smaller for $w=4.0 \mu \mathrm{m}$ than for $w=3.5 \mu \mathrm{m}$. The two shoulders, marked by the two black arrows in fig. 3, suggest that the optical profile of $w=4.0 \mu \mathrm{m}$ does not exhibit four but six peaks. For $w=3.0 \mu \mathrm{m}$ and $w=2.5 \mu \mathrm{m}$ stripes, we found a profile featuring three peaks with $p$ respectively equal to $580 \mathrm{~nm}$ and $480 \mathrm{~nm}$. If the width is reduced to $w=2.0 \mu \mathrm{m}$, only two peaks arise. Finally, the optical response shows a large central peak flanked by two small amplitude oscillations located close to the edges of the stripe for $w=1.5 \mu \mathrm{m}$.

To explain the structure of the MS near-field optical response, one could first invoke a standing wave pattern originating from the interference of SP propagating at the stripe surface along symmetric zigzag paths(Fig. 4). If $k^{M S}$ denotes wave vector of the SP propagating along the stripe, the components $k_{x}^{M S}$ and $k_{y}^{M S}$ are respectively given by $k_{x}^{M S}=k^{M S}$ $\cos \delta$ and $k_{y}^{M S}=k^{M S} \sin \delta$, where $\delta$ stands for the angle of incidence of the SP on the edges of the stripe. The component $\mathrm{k}_{x}^{M S}$ can also be written as $k_{x}^{M S}=\frac{\pi}{p}$ if $p$ denotes the period of the interferences observed in the optical profiles. Because the excitation of the stripes is achieved using a surface plasmon launched on the thin film area, we can assume that $k_{y}^{M S}=k_{S P}$ where $k_{S P}$ is the wave-vector of the SP propagating at the surface of a thin film. With this assumption, the angle $\delta$ is given by: 


$$
\delta=\arctan \frac{p \times k_{S P}}{\pi}
$$

The angle of incidence $\theta_{S P}$ allowing the excitation of the metal thin film SP is given by the minimum reflection of a TM polarized collimated beam illuminating a metal thin film in the Kretschmann-Raether configuration. The metal thin film SP wave-vector can be computed from $k_{S P}=n k_{0} \sin \theta_{S P}$ where $n k_{0}$ is the wave-vector of the incident light in the substrate supporting the thin film. For a frequency corresponding to a wavelength in vacuum of $800 \mathrm{~nm}$ and a gold film with a thickness of $52 \mathrm{~nm}$, we found the experimental value of $k_{S P}$ to be $7.90 \mu \mathrm{m}^{-1}$. From this value and the values of the periodicity $p$ measured over the various stripes, we obtain an angle $\delta$ ranging from $47^{\circ}$ to $55^{\circ}$. Then, if we suppose that the SP propagation along a stripe relies on reflections at edges, it's a simple matter to show that, for the stripes we consider, the SP should undergo several tens of reflections in order to propagate a few tens of microns. Even if a step discontinuity can reflect efficiently a surface plasmon, previous works established that the reflection of SP on an abrupt edge of a thin film is far from being total $[14-16,9]$. Thus, such a kind of propagation would obviously lead to a dramatic damping of the SP amplitude along the stripe axis. Since we find experimentally that the damping of a MS plasmon mode is comparable to those of a SP launched on an extended thin film, we conclude that SP guiding along MS does not rely on reflections at the MS edges. In other words, the near-field intensity distributions shown in Fig. 3 are not standing wave patterns as in dielectric waveguides, but a genuine property of the MS surface plasmon modes.

In order to gain more insight in the role played by the edges of the stripes, we consider the sample shown in figure 5(a). The sample is a rectangular gold thin film area (thickness $=50 \mathrm{~nm}$ ) with a surface of $90 \times 40 \mu \mathrm{m}^{2}$. The PSTM image of figure $5(\mathrm{~b})$ has been recorded when the upper right corner of the thin film area is illuminated by the incident spot. The average angle of incidence of the focused beam was taken equal to $\theta_{S P}$ and the plane of incidence was adjusted to be parallel to the $y$ axis. With these illumination conditions, a surface plasmon is locally excited on the upper part of the thin film and propagates towards 
the negatives values of $y$. As in the case of the stripes, we observe in the PSTM image, a series of longitudinal oscillations (parallel to the $y$ axis) with a period $p=480 \mathrm{~nm}$. These oscillations are even more clearly visible in the cross-cut displays in figure 5(d). The same kind of features are observed if the focused beam is replaced by a collimated one (figure $5(\mathrm{c})$ ). In this case, the plasmon is excited over the whole surface of the thin film. The interference of the incident SP and the SP back-reflected by the edge of the thin film perpendicular to the plane of incidence leads to a standing surface-wave pattern parallel to the $x$ axis. For an incident $\mathrm{SP}$ with a positive $x$ wave-vector component, a reflection on the thin film edge parallel to the $y$ axis could also explained the longitudinal oscillations. However, as in the case of the MS, the angle of incidence $\delta$ of the SP on the edge should be around $50^{\circ}$ in order to create a standing wave-pattern with a period of $480 \mathrm{~nm}$. Since the plane of incidence was adjusted to be parallel to the $y$ axis, such an angle of incidence $\delta$ is unrealistic. In addition, one can see on figure 5(c) that the amplitude of the longitudinal oscillations is damped over a typical distance of about two microns while no damping is visible for the standing wave pattern parallel to the $x$ axis. On the basis of these observations, we conclude that the longitudinal oscillations are not a standing wave pattern but corresponds probably to the near-field distribution of a mode supported by a boundary between a semi-infinite metal thin film and a dielectric medium. Recent numerical results [2,17] demonstrate that highly confined edge modes (called "corner modes" in Refs [2,17]) are expected at the step discontinuity of a metal thin film and a dielectric medium. However, both the confinement and the field enhancement of these type of modes are very sensitive to the geometry of the edge [10]. This is probably because the lithographic technique does not allow to achieve edges with a radius of curvature smaller than a few tens of nanometers that we do not observe genuine edges modes but rather SP modes that characterize the presence of the thin film boundary. Note that similar type of oscillating "boundary modes" with a low degree of localization have been described in the context of surface phonons at the transverse boundary between two semi-conducting medium [18]. From the results obtained for the extended thin film boundary, it is now easy to convince oneself that the stripe modes are coupled boundary 
and interface modes. Indeed, the optical profiles of each stripe (Fig. 3) is comprised of a gaussian-like intensity distribution (associated with a finite width interface mode) modulated by the longitudinal oscillations of the two boundary modes supported by each lateral boundary of the MS. Prior to a more complete analysis of the MS plasmon modes, it is necessary to know first if the field distributions we observe are related to a monomode or multimode excitation.

\section{MULTIMODE OR MONOMODE EXCITATION?}

At this stage, due to the focused nature of the incident beam, we can not unambiguously determine if the measured intensity distribution is due to the excitation of only one or several MS surface plasmon modes. A definitive resolution to this question is not possible in the absence of the width-dependent dispersion relations of SP modes supported by MS bounded by different dielectric media such that a large contrast of the index refraction arises between the substrate (glass in our case) and the external medium (air in our case) [17]. However, two arguments favor the thesis of the excitation of only one MS surface plasmon mode.

First, as mentioned above, the stripes are not directly excited by the focused beam but by the SP launched on the extended thin film area. The resonance linewidth of this launched SP that reaches the stripe imposes a narrow range of wave-vector components $k_{\|}$which are parallel to the surface. This suggests that the SP launching process has filtered a single $k_{\|}=k_{S P}$ out of those provided by the numerical aperture of the focusing lens. In other words, the extended thin film area acts as a narrow band-pass $k_{\|}$filter selecting $k_{S P}$. Such a technique excites only the MS surface plasmon modes whose dispersion relation cut the line $k_{\|}=k_{S P}$ at the incident frequency $\omega_{0}=2 \pi c / \lambda$ ( $c$ being the speed of light in vacuum). The excitation of several modes would imply that the dispersion relations of the MS surface plasmon modes display several branches in the vicinity of the point $\left(\omega_{0}, k_{S P}\right)$. In this case, the illumination conditions should lead to the excitation of the mode whose phase constant is the closest to $k_{S P}$ at the incident frequency. 
A second argument favoring the excitation of only one MS surface plasmon mode relies on the invariance of the transverse modal structure along the longitudinal axis of the stripe. Let us consider the PSTM image of the junction between the extended thin film area and the $w=2.5 \mu \mathrm{m}$ MS (figure 6(b)). The SP launched on the extended thin film area is visible in the upper part of this image. The characteristic three peaks transverse profile is established above the MS in the very first few micrometers after the junction to the extended thin film area. Note that no spatial transient is visible around this junction zone. This suggests that coupling the launched SP to the MS surface plasmon mode does not induce any significant radiation losses [19]. One can also observe that the three peaks structure remains stable all along the stripe and is similar to those recorded further away from the junction zone in figure $2(\mathrm{~d})$. If we assume a multi-modal excitation of the MS, at a constant height $z_{0}$ above the stripe, the electric field of the SP propagating along the MS reads:

$$
\vec{E}\left(x, y, z_{0}\right)=\sum_{m} \vec{E}^{(m)}\left(x, z_{0}\right) \exp \left(-\alpha^{(m)}+i \beta^{(m)}\right) y
$$

where $\vec{E}^{(m)}\left(x, z_{0}\right), \alpha^{(m)}$ and $\beta^{(m)}$ denote respectively the amplitude, the attenuation constant and the phase constant of each MS surface plasmon mode. In the illumination conditions detailed above, the simultaneous excitation of several MS modes implies that their phase constants should be equal (or at least very close) to $k_{S P}$, so that the expression of the electric field becomes:

$$
\vec{E}\left(x, y, z_{0}\right)=\sum_{m} \vec{E}^{(m)}\left(x, z_{0}\right) \exp \left(-\alpha^{(m)}+i k_{S P}\right) y
$$

The observed invariance of the transverse profile of the near-field intensity distribution along $y$ requires also the equality of the attenuation constants of all the modes simultaneously excited. Indeed, the relative weights of each mode involved in the above expansion of the total field must be kept constant as a function of $y$ in order to agree with the observed invariance. Thus, it turns out that the hypothesis of a multi-modal excitation implies the equality of both the phase and attenuation constants of all the MS surface plasmon modes which are simultaneously excited. Since, these two constants are usually not equal from 
one MS surface plasmon mode to the other $[2,17]$, we conclude that there is a very high probability that the experimental images show the intensity distribution of only one mode for each MS.

Up to now, the metal stripes have been excited by coupling with a SP locally launched on an extended thin film area. With the aim of checking the influence of the excitation conditions on the near-field intensity distribution, we consider now a direct illumination of a gold stripe having a width of $3.25 \mu \mathrm{m}$. Figure 7 (a) shows a topographic image of this stripe and the location of the incident spot. The average angle of incidence of the focused beam was taken equal to $\theta_{S P}$ and the plane of incidence was adjusted to be parallel to the $y$ axis. Both the PSTM image (figure $7(\mathrm{~b})$ ) and the optical profile displays in figure 7(c) show a four peaks intensity distribution in agreement with the optical response of the MS having approximately the same width ( $\mathrm{W}=3.5 \mu \mathrm{m}$ in fig. 3). Except for the period of the peaks which is now $500 \mathrm{~nm}$ instead of $580 \mathrm{~nm}$, the optical profiles obtained for $\mathrm{W}=3.5 \mu \mathrm{m}$ and $\mathrm{W}=3.25 \mu \mathrm{m}$ are very similar. This observation leads to the conclusion that the direct illumination of a MS does not allow the excitation of a SP modes different from those excited by coupling with a thin film SP. It is worth to notice that this result arises in spite of the wide range of $k_{\|}$incident on the MS when it is directly illuminated by the focused beam. It turns out that even if the dispersion curve of several MS modes cut the line $\omega=\omega_{0}$ in the range of $k_{\|}$defined by the angular width of the incident beam, only one mode is efficiently excited with this type of illumination. Since, it now clear that the field distributions we observe are related to a single mode excitation, we discuss in detail the characteristics of this mode in the next section.

\section{INSIGHT IN THE DISPERSION RELATIONS OF METAL STRIPE SP}

The numerical results of references [17] demonstrate that the coupling between SP modes supported respectively by the substrate/metal and metal/superstrate interfaces can make the field distribution of MS modes very sensitive to the thickness of the metal film. This 
property is true for a symmetric MS (optical index of the substrate $\left(\mathrm{n}_{\text {sub }}\right)$ and superstrate $\left(\mathrm{n}_{\text {sup }}\right)$ are equal $)$ but also for asymmetric system $\left(\mathrm{n}_{\text {sub }} \neq \mathrm{n}_{\text {sup }}\right)$ even in the case of a large asymmetry. In order to check the behavior of the MS modes with respect to the thickness of the metal film, the sample shown in figure 8(a) was fabricated. This sample is comprised of a MS with a width of $3.25 \mu \mathrm{m}$ connected to a large thin film area. The main difference between this sample and the previous ones is the thickness of the gold film measured at $120 \mathrm{~nm}$ by AFM microscopy. We found experimentally this thickness to be the largest that allows the excitation of a surface plasmon at the gold/air interface of the thin film in the KretschmannRaether configuration using our focused beam. Figure 8(b) shows the PSTM image recorded over the junction area defined by the white dashed square drawn in figure 8(a). A neat four peaks structure is visible over stripe. The structure can be observed even after a propagation of more than 20 microns (Fig. 8(c)). The optical profile obtained for the "thick" MS and the "thin" MS previously studied in figure 7 are surimposed in figure $8(\mathrm{~d})$. Because the two profiles are qualitatively similar, we conclude that the thickness of the film does not play a key role in the field distribution of the MS plasmon modes. Thus, the modes we observe are not created by a coupling of SPs supported by the upper and lower interface of the MS. Note that, because the field enhancement of a SP excited at the surface of a "thick" film is lower than for a "thin" film [1], the optical response of the "thick" MS (solid line in fig. 8(d)) exhibits a lower confinement along the $x$ axis than the response of the corresponding "thin" MS (dashed line in fig. 8(d)).

When excited by coupling with a SP launched on the thin film area, the electromagnetic field incident on a MS is mostly localized at the upper (metal/air) interface. The main component of the electric field associated with a thin film SP is known to be the component perpendicular to the substrate surface ( $z$ direction in our case, see fig. reffig4). If the thin film SP propagates along the $y$ axis, the $z$ component of its electric field is then symmetric with respect to the $y z$ plane. Thus, according to the nomenclature defined in reference [17], such a field should allow an efficient excitation of a " $S S_{b}^{n}$ " type MS mode, where $n$ labelled the number of extremum along the $x$ axis of the MS mode electric field $z$ component. As we 
mentioned previously, the cross-sections of the MS we consider are not perfectly rectangular such that it is difficult to push further the analysis of our experimental results by direct comparison with the numerical computations of reference [17]. In particular, the number of peaks we observe in the optical profiles of the MS modes can not be interpret as the value of $n$ previously defined. Nevertheless, one can get some insight in the width-dependent dispersion relation of the MS plasmon modes from our experimental observations.

The near-field optical profiles (figure 3) show that, for a fixed frequency, decreasing the MS width may have two main consequences on the SP modes intensity distribution. First, the distance between the peaks can be reduced while the number of peaks is kept constant as, for example, in the transition from $w=3.0 \mu \mathrm{m}$ to $w=2.5 \mu \mathrm{m}$. Second, if the width is further reduced below a critical width, the number of peaks is lowered, as, for examples, in the transition from $w=3.5 \mu \mathrm{m}$ to $w=3.0 \mu \mathrm{m}$ or from $w=2.5 \mu \mathrm{m}$ to $w=2.0 \mu \mathrm{m}$. Both behaviors suggest that, at the frequency $\omega_{0}$, a mode featuring a given number of peaks may occur in some range of widths. Below some critical width, the dispersion curve of a given mode (with a characteristic number of peaks) probably shifts toward higher energy such that it is no more possible to excite it at the frequency we use. Thus, below this critical width, the number of peaks in the MS plasmon mode profile is lowered.

This reasoning seems to be refuted by the transition from $w=2.0 \mu \mathrm{m}$ to $w=1.5 \mu \mathrm{m}$. In this case, the number of peaks in the transverse modal profile increase from two to three. However, in the near-field optical transverse profile of the $1.5 \mu \mathrm{m}$ wide stripe, the amplitude of the central peak is much larger than those of the two other peaks which are located very close to the edges of the stripe. This localization suggest that such peaks might be related to the strong coupling of the two boundary modes. This assumption is difficult to verify at this stage but would at least restore the consistency of the reduction of the number of peaks associated with the transverse profile of the SP mode when decreasing the width of the MS. Contrasting with the SP modes confined within the width of the stripes observed for $w>1.5 \mu \mathrm{m}$, the intensity decaying far away outside the stripe provides another striking feature of this width $w=1.5 \mu \mathrm{m}$. In analogy with dielectric waveguides, this somewhat 
softer confinement could indicate that $1.5 \mu \mathrm{m}$ is close to the "cut-off width" of this mode.

\section{CONCLUSION}

In summary, we have used a PSTM to observe the near-field intensity distribution of plasmon modes propagating on gold stripes featuring widths in the micrometer range. The illumination conditions allowed to excite the stripes by coupling with a surface plasmon polariton launched on an extended thin film area. For a fixed frequency corresponding to a wavelength in vacuum of $800 \mathrm{~nm}$, the PSTM images show that the MS surface plasmon modes remain well confined within the stripe widths. If the MS width is not too large, the near-field optical transverse profiles of the MS surface plasmon modes exhibit an intensity distribution showing periodic oscillations along the transverse axis. By comparing the field distribution observed over a MS excited either by direct illumination with the incident focused spot or by coupling with a SP launched on an extended thin film, we conclude that the features of the MS optical profiles are probably related to the excitation of a single MS mode. From the PSTM observation of a single metal thin film step discontinuity, we show that the transverse features in the field distribution of the MS plasmon mode are not standing wave patterns but are related to oscillating modes characterizing the existence of an abrupt boundary between a metal thin film and a dielectric medium. These observations lead to the conclusion that the propagation of SP modes along MS does not rely on reflections on the lateral edges of the metal stripe. Thus, the propagation of a SP along a MS is fundamentally different from the propagation of guided modes in standard dielectric waveguides. One can then anticipate specific guiding properties of MS. The similarity of the intensity distributions recorded over MS having the same width but different thickness demonstrates that the field of the MS modes is mostly localized at the upper interface (metal/air). Even if the frequency dependence of the metal dielectric function prevents a straightforward extrapolation of the MS optical properties to other frequencies, the experimental results reported here, at a fixed frequency and for different widths, provide an useful insight in the dispersion relations of 
MS surface plasmons.

\section{ACKNOWLEDGMENTS}

This work was supported by the Regional Council of Burgundy (ARCEN project) and by the French CNRS (STIC: EPML 20). 


\section{REFERENCES}

[1] H. Raether, Surface Plasmons, Springer Tracts in Modern Physics, Vol 111 (Springer, Berlin, 1988).

[2] P. Berini, Phys. Rev. B, 61, 10484 (2000).

[3] P. Berini, Optics Express, 7, 329 (2000).

[4] R. Charbonneau, P. Berini, E. Berolo, E. Lisicka-Shrzek, Optics Lett. 25, 844 (2000).

[5] B. Lamprecht et al, Appl. Phys. Lett., 79, 51, (2001).

[6] H. Ditlbacher et al, Appl. Phys. Lett., 81, 1762, (2002).

[7] R. C. Reddick, R. J. Warmack, T. L. Ferrell, Phys. Rev. B, 39, 767 (1989).

[8] J. C. Weeber et al, Phys. Rev. B, 64, 045411, (2001).

[9] See for example figures 3(b) and 3(c) of reference [8]

[10] J. P. Kottmann, O.J.F. Martin, D.R. Smith and S. Schultz, Phys. Rev. B, 64, 235402, (2001).

[11] A. Dereux, E. Devaux, J. C. Weeber, J. P. Goudonnet, C. Girard, J. Microscopy 202,320 (2001).

[12] P. Dawson, F. de Fornel, J. P. Goudonnet, Phys. Rev. Lett., 72, 2927 (1994).

[13], R. Quidant et al, Phys. Rev. E, 64, 066607, (2001).

[14] J. A. Sánchez-gil, Appl. Phys. Lett., 73, 3509, (1998).

[15] R. F. Wallis, A. A. Maradudin, G. I. Stegeman, Appl. Phys. Lett., 42, 764, (1983).

[16] T. P. Shen, R.F. Wallis, A. A. Maradudin, G.I. Stegeman, Appl. Optics, 23, 607, (1984).

[17] P. Berini, Phys. Rev. B, 63, 125417 (2001).

[18] Ph. Lambin, T. Laloyaux, A. A. Lucas and J. P. Vigneron, Phys. Rev. B, 35, 5621 
(1987).

[19] A. W. Snyder and J. D. Love, Optical Waveguide Theory, Chapman \& Hall, London, (1983). 


\section{FIGURES}
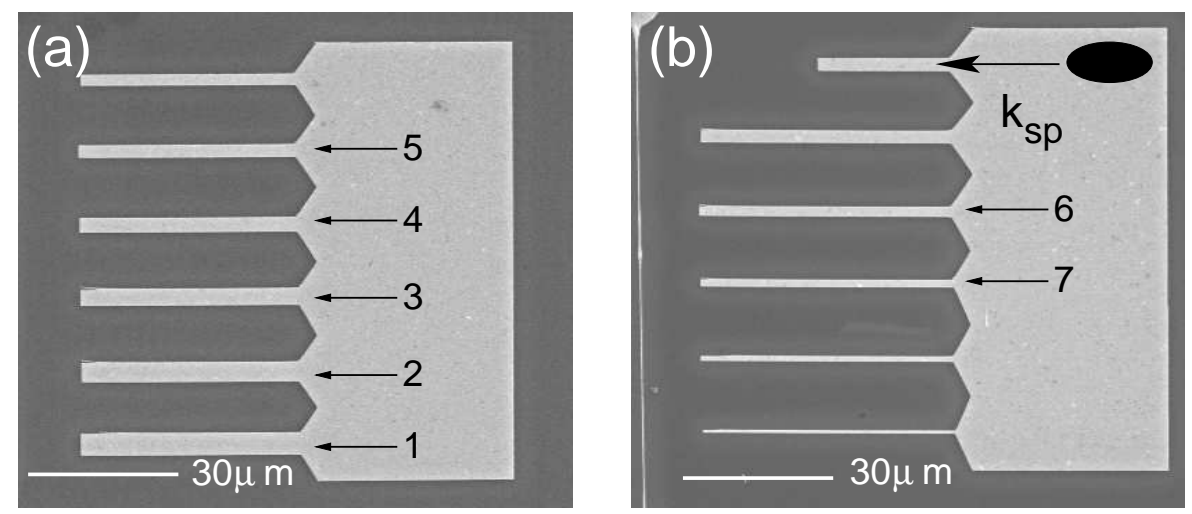

FIG. 1. Scanning electron microscope images of $55 \mathrm{~nm}$ thick gold stripes. The widths of the stripes, labelled from (1) to (7), ranges from 4.5 to $1.5 \mu \mathrm{m}$ by step of $0.5 \mu \mathrm{m}$. A focused laser beam $(\lambda=800 \mathrm{~nm})$ is incident from the substrate at an angle suitable to excite SP on the extended thin film. An example of the location of the resulting spot is schematically shown in the upper part of fig. 1(b). 

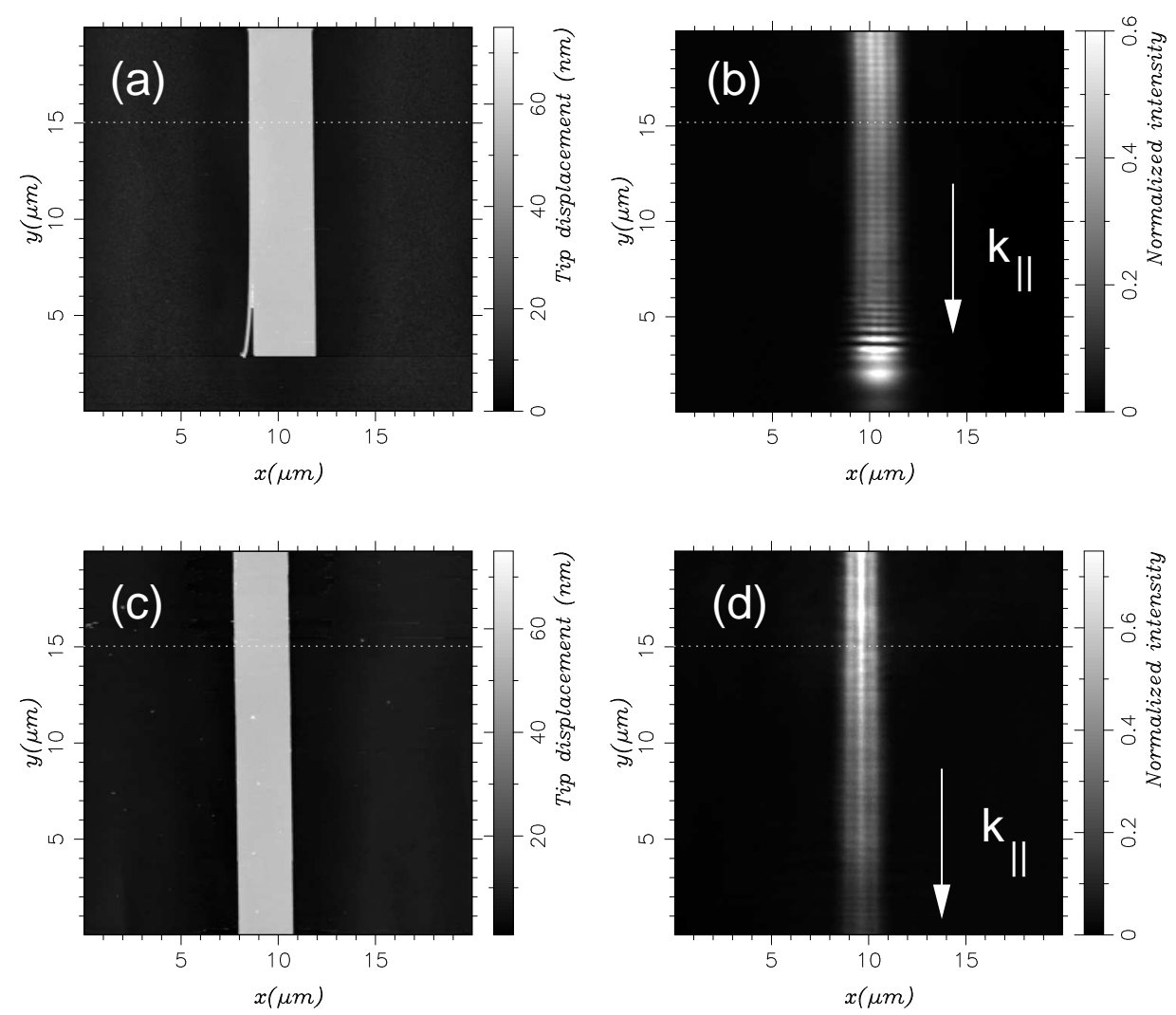

FIG. 2. AFM ((a) and (c)) and PSTM ((b) and (d)) images of two stripes: $w=3.5 \mu \mathrm{m}$ in (a) and (b) while $w=2.5 \mu \mathrm{m}$ in (c) and (d). The intensity scales of the PSTM images are normalized independently of each other: for each sample, the unit intensity is defined as the highest intensity detected above the stripe. For the sake of better visualisation, the scale has been saturated to 0.6 in (b) and to 0.75 in (d). 

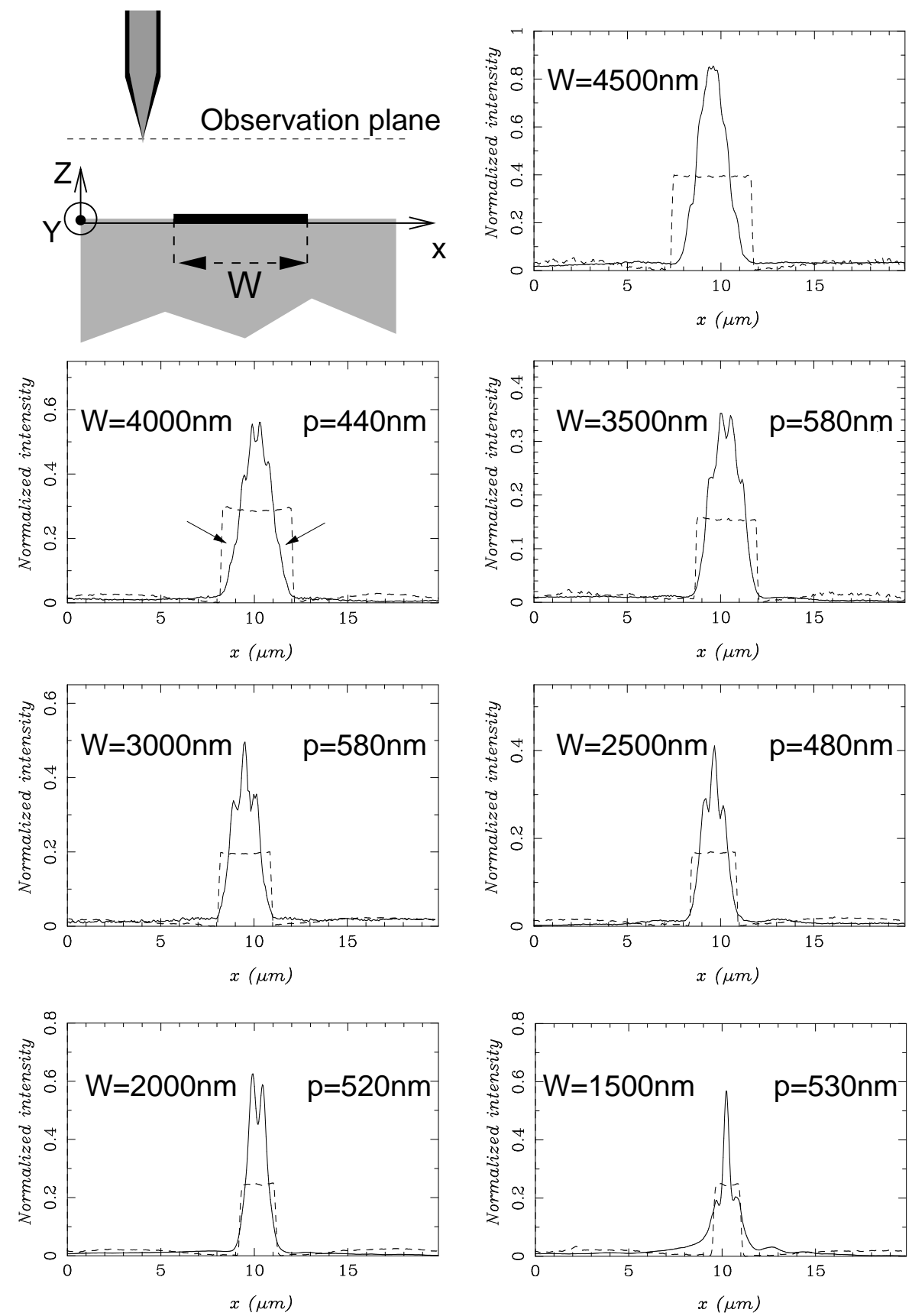

FIG. 3. Over the various stripes, cross-cuts of the near-field intensity distribution recorded at a constant height. The dashed lines are guides to the eyes indicating the stripes widths (see text). The values of $p$ give the distances between the peaks. 


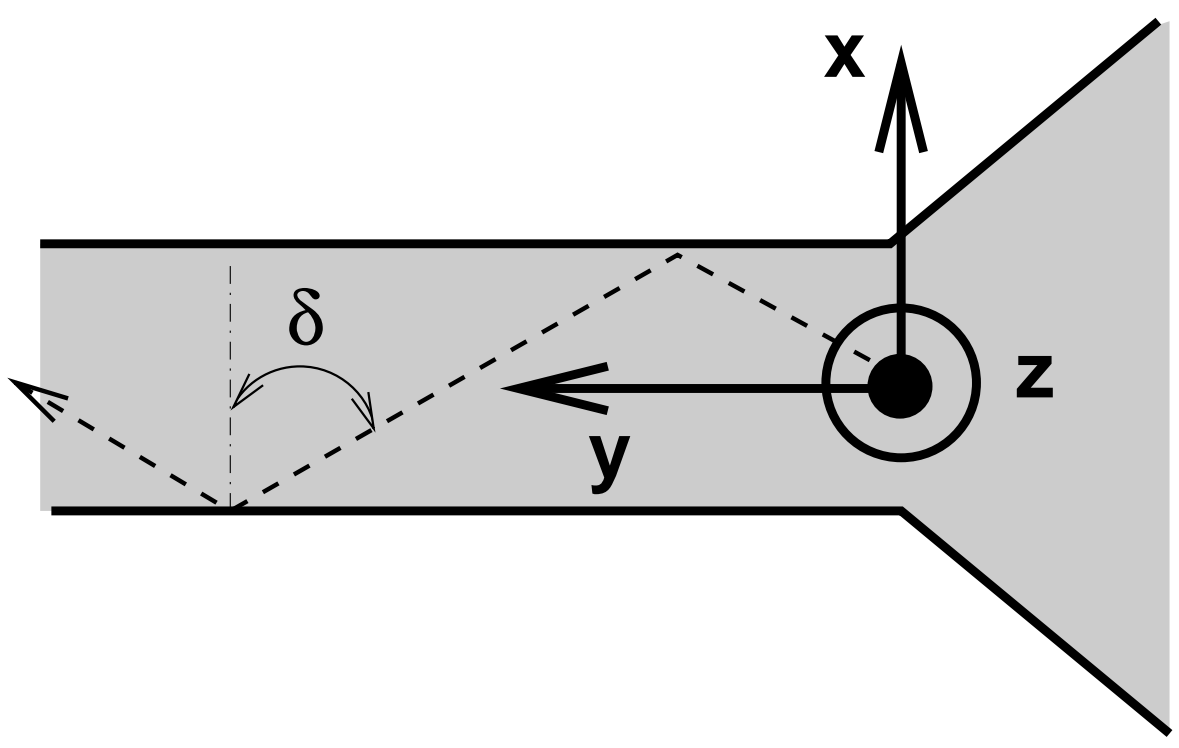

FIG. 4. Schematic view of a SP multi-reflection on the edges of a metal stripe 

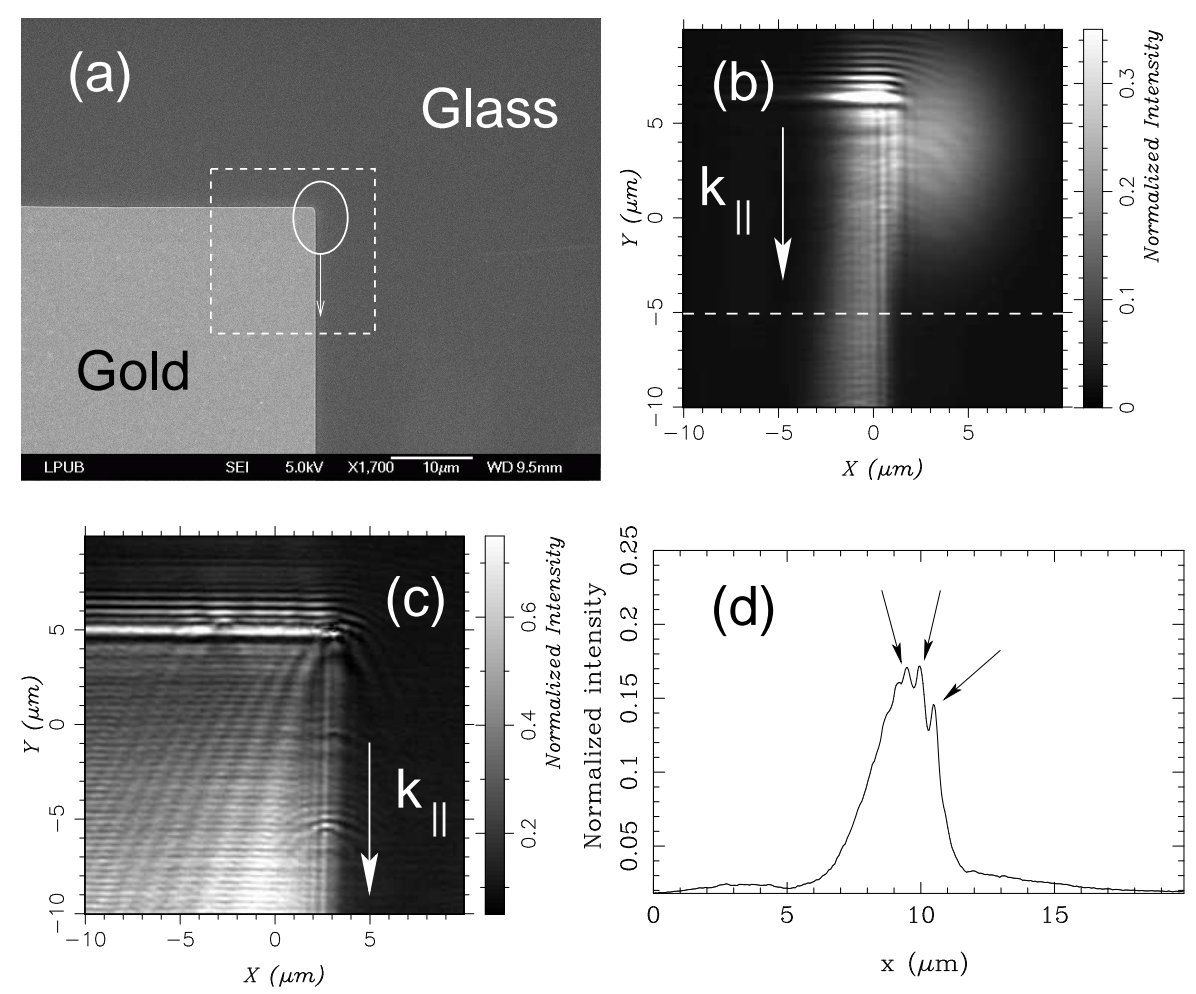

FIG. 5. (a) Scanning electron microscope (SEM) image of a step discontinuity of a gold thin film. (b) PSTM image recorded over the surface defined by the white dashed perimeter drawn in (a). The upper right corner of the gold thin film area is excited by an incident focused spot. The location of the incident spot is schematically shown on (a). The plane of incidence is parallel to the $y$ axis .(c) Same as (b) except that the incident beam is not focused anymore but collimated (spot diameter $600 \mu \mathrm{m}$ ). A surface plasmon is excited over the whole surface of the gold thin film. (d) Cross-cut of the image (b) taken along the white dashed line. 

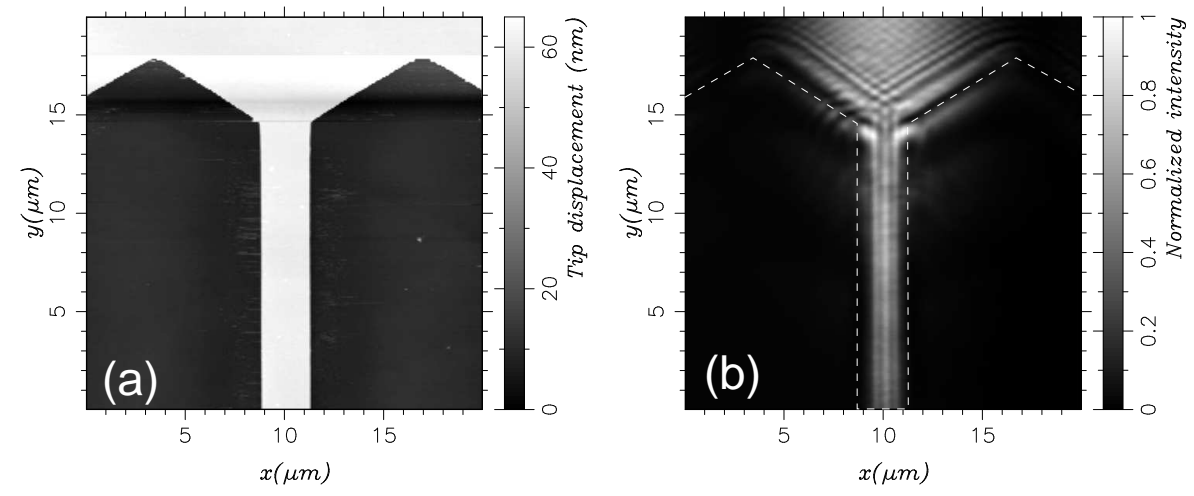

FIG. 6. AFM (a) and PSTM (b) images of the junction between the extended thin film and the $2.5 \mu \mathrm{m}$-wide stripe. 

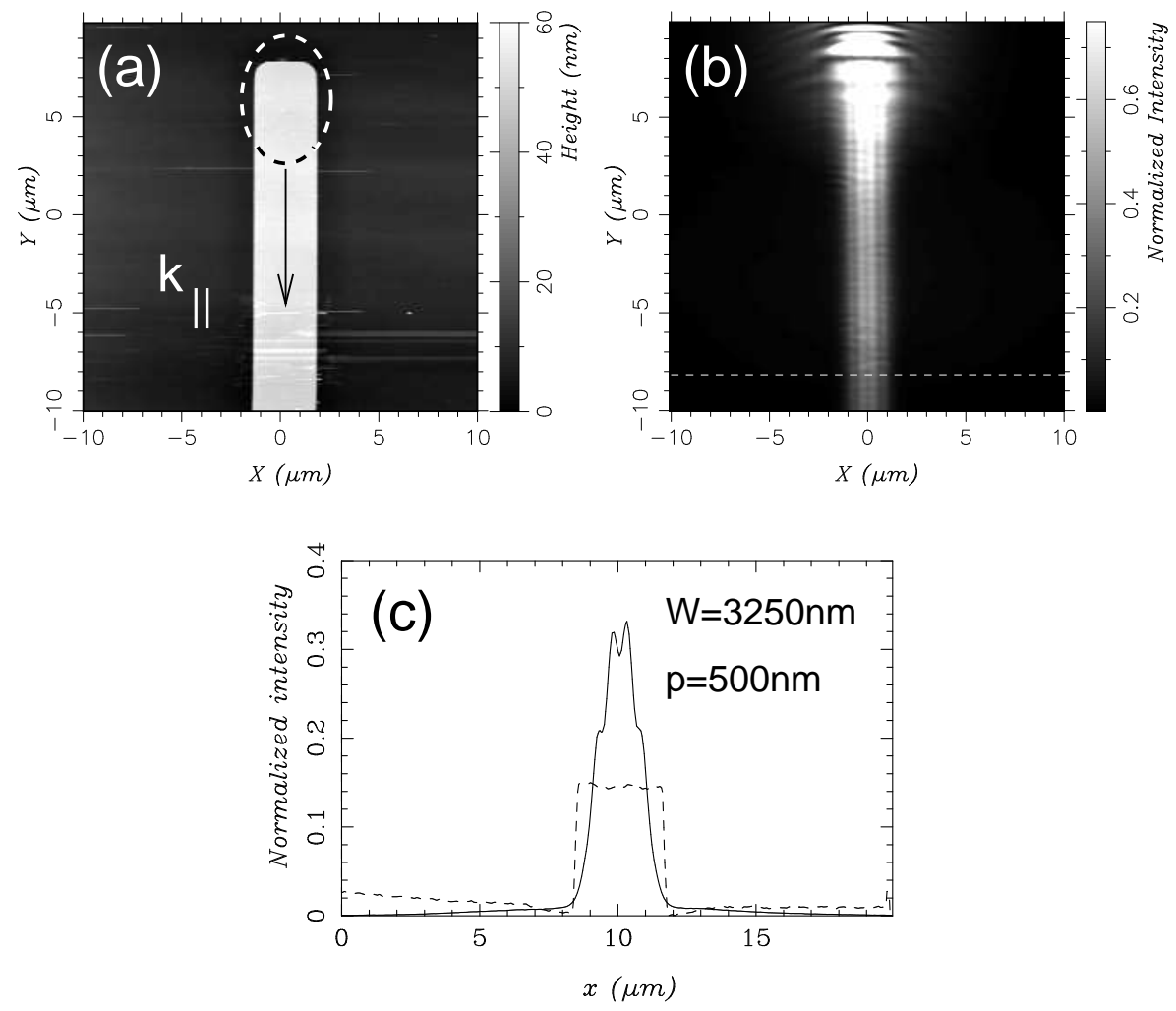

FIG. 7. (a) Topographic image of a MS with a width $\mathrm{W}=3.25 \mu \mathrm{m}$ and thickness $\mathrm{t}=55 \mathrm{~nm}$. (b) PSTM image recorded when the input end of the MS is directly illuminated by the incident spot. The average angle of incidence is equal to $\theta_{S P}$ and the plane of incidence is parallel to the $y$ axis. (c) Cross-cut of (b) taken along the white dashed line. The period of the longitudinal oscillations is $500 \mathrm{~nm}$. 

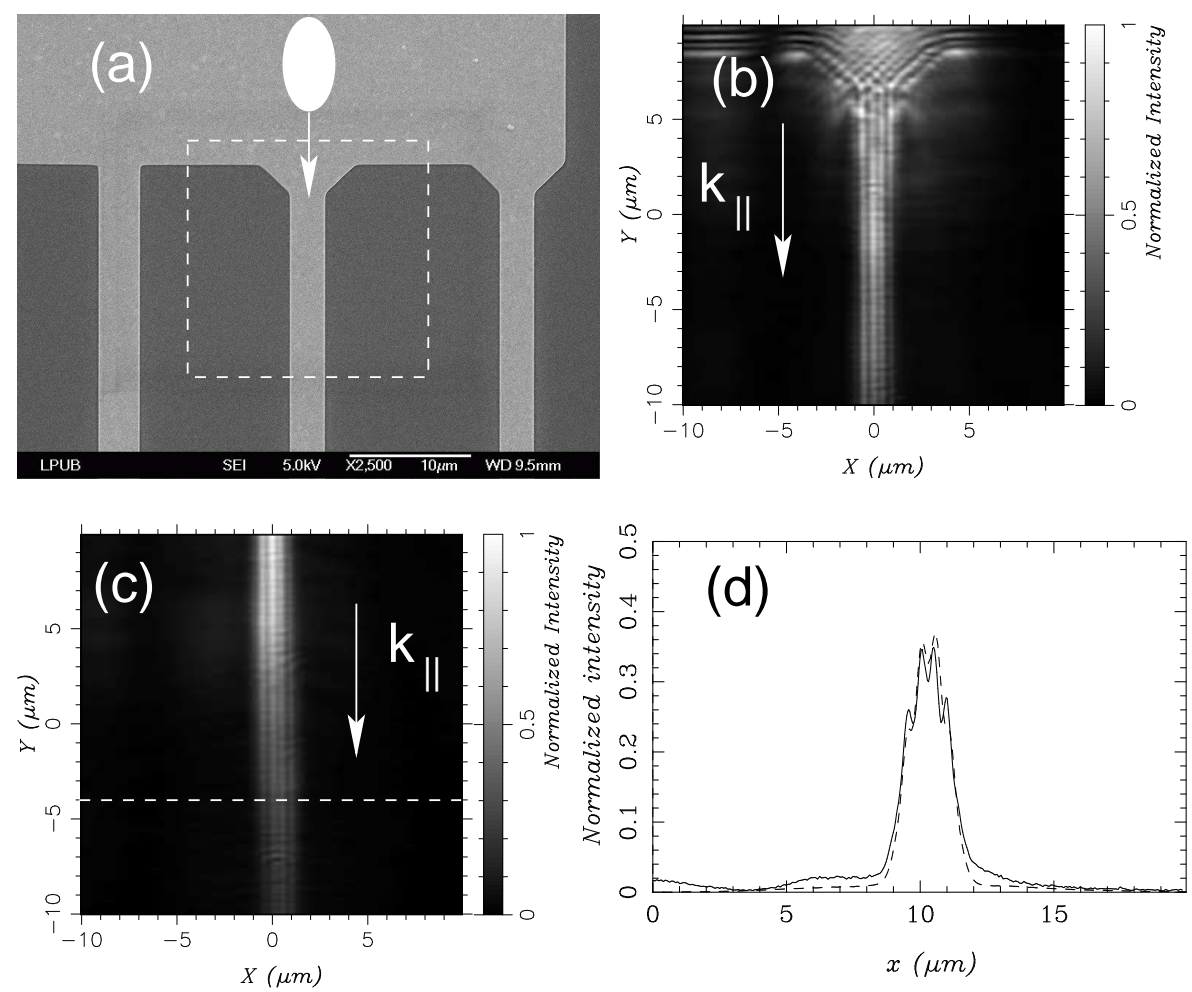

FIG. 8. (a) SEM image of the "thick" sample.(Thickness=120nm). (b) PSTM image recorded over the junction zone defined by the white dashed square drawn on (a). The width of the MS is $\mathrm{W}=3.25 \mu \mathrm{m}$. The excitation of the MS is achieved by coupling with a SP locally launched by the incident focused beam. The incident spot is localized on the extended thin film area. (c) Same as (b) except that the image is taken after a propagation of MS mode over more than $20 \mu \mathrm{m}$. (d) Solid line: Cross-cut of image (c) taken along the white dashed line. Dashed line: Optical profile shown in fig. $7(\mathrm{c})($ Thickness $=55 \mathrm{~nm})$. Note that the amplitude of this profile has been adjusted for the sake of easier comparison between the optical responses of the "thick" and the "thin" MS. 